\title{
THE APPROXIMATION OF THE EXACT BOUNDARY CONDITIONS AT AN ARTIFICIAL BOUNDARY FOR LINEAR ELASTIC EQUATIONS AND ITS APPLICATION
}

\author{
HOUDE HAN AND XIAONAN WU
}

\begin{abstract}
The exterior boundary value problems of linear elastic equations are considered. A sequence of approximations to the exact boundary conditions at an artificial boundary is given. Then the original problem is reduced to a boundary value problem on a bounded domain. Furthermore, a finite element approximation of this problem and optimal error estimates are obtained. Finally, a numerical example shows the effectiveness of this method.
\end{abstract}

\section{INTRODUCTION}

Many boundary value problems of partial differential equations arising in practical applications are given on unbounded domains, such as coupling of structures with foundation and fluid flow around obstacles. In finding the numerical solutions of these problems, it is often difficult to use the classical finite element or finite difference method. In engineering, the usual method is to introduce an artificial boundary and cut off the unbounded part of the domain and to set up an artificial boundary condition at the artificial boundary of the remaining bounded domain. For example, the Dirichlet condition and Neumann condition are often used for elliptic partial differential equations. In general, this artificial boundary condition at the artificial boundary is only a rough approximation of the exact boundary condition. Hence, the remaining bounded domain must be quite large when high accuracy is required. For such large domains, it is still difficult to compute the numerical solution.

In 1985, we found the exact boundary conditions at an artificial boundary for the Laplace equation as a model equation [7]. Moreover, a sequence of approximations to the exact boundary condition at the artificial boundary was given, and we reduced the original exterior problem to an equivalent (or approximate) boundary value problem on a bounded domain with integral boundary conditions. Then we solved the approximate boundary value problem on the bounded domain by a finite element method. An optimal error estimate of the finite element approximate solution was obtained and a numerical example showed the effectiveness of this method.

Received by the editor July 26, 1990.

1991 Mathematics Subject Classification. Primary 35A35, 35A40, 65N99.

Key words and phrases. Unbounded domains, artificial boundaries, approximate boundary conditions at an artificial boundary.

The first author was supported in part by the National Natural Science Foundation of China. 
Boundary value problems on unbounded domains have been studied for many years. For example, in 1982, Goldstein [3] studied Helmholtz-type equations. The problem was replaced by a boundary value problem on a fixed bounded domain. The behavior of the solution near infinity is incorporated in a nonlocal boundary condition. In 1984, Feng [4] studied asymptotic radiation conditions for the reduced wave equation; in 1986, Hagstrom and Keller [5] studied the exact boundary condition at an artificial boundary for partial differential equations in cylinders. Shortly thereafter, they used this technique to solve nonlinear problems of both elliptic and parabolic type [6]. This technique is a rather natural extension of related work on ordinary differential equations over infinite intervals by Keller [9], Jepson and Keller [8], and Lentini and Keller [11]. In 1988, Lenoir and Tounsi [10] studied the various convergence properties of the localized finite element method for the two-dimensional sea-keeping problem.

In this paper we show how this technique applies to the exterior problem for the linear elastic equations and obtain its finite element approximation on a bounded domain. An optimal error estimate of the finite element approximate solution is given; moreover, a numerical example shows this technique to be very effective.

\section{THE EXACT AND APPROXIMATE BOUNDARY CONDITIONS AT AN ARTIFICIAL BOUNDARY}

Let $\Gamma_{i}$ be a bounded, simply closed curve in $R^{2}$, and let $\Omega$ be the unbounded domain with boundary $\Gamma_{i}$. Consider the following exterior boundary value problem:

$$
\begin{aligned}
&-\mu \Delta u-(\lambda+\mu) \frac{\partial}{\partial x}\left(\frac{\partial u}{\partial x}+\frac{\partial v}{\partial y}\right)=f_{1} \quad \text { in } \Omega, \\
&-\mu \Delta v-(\lambda+\mu) \frac{\partial}{\partial y}\left(\frac{\partial u}{\partial x}+\frac{\partial v}{\partial y}\right)=f_{2} \quad \text { in } \Omega, \\
& u=0 \text { on } \Gamma_{i}, \\
& v=0 \text { on } \Gamma_{i}, \\
& u, v \text { are bounded when } r=\left(x^{2}+y^{2}\right)^{1 / 2} \rightarrow+\infty,
\end{aligned}
$$

where $(u, v)$ is the displacement, $\lambda, \mu>0$ are the Lamé constants, and $\left(f_{1}, f_{2}\right)$ is the density of the applied body force, the support of which is compact.

This problem is defined on an unbounded domain $\Omega$. The usual method engineers use is to draw a circumference $\Gamma_{e}$ with radius $R$. Then $\Omega$ is divided into two parts; the bounded part and the unbounded part are denoted by $\Omega_{i}$ and $\Omega_{e}$ (see Figure 1). Furthermore, suppose that the support of $\left(f_{1}, f_{2}\right)$ is in $\Omega_{i}$. If a certain boundary condition on the artificial boundary $\Gamma_{e}$ is given, then we could solve the problem (1)-(4) on the bounded domain $\Omega_{i}$. The goal of this section is to derive the exact and an approximate boundary condition for the solution of problem (1)-(4) on $\Gamma_{e}$.

We now consider the boundary value problem of linear elastic equations on 


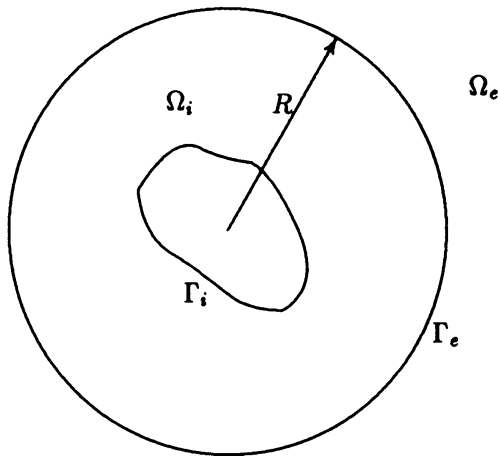

FIGURE 1

the unbounded domain $\Omega_{e}$ with boundary $\Gamma_{e}$ :

$$
\begin{aligned}
-\mu \Delta u-(\lambda+u) \frac{\partial}{\partial x}\left(\frac{\partial u}{\partial x}+\frac{\partial v}{\partial y}\right) & =0 \text { in } \Omega_{e}, \\
-\mu \Delta v-(\lambda+\mu) \frac{\partial}{\partial y}\left(\frac{\partial u}{\partial x}+\frac{\partial v}{\partial y}\right) & =0 \text { in } \Omega_{e}, \\
\left.u\right|_{\Gamma_{e}} & =u(R, \theta), \\
\left.v\right|_{\Gamma_{e}} & =v(R, \theta),
\end{aligned}
$$

$u, v$ are bounded when $r \rightarrow+\infty$.

We know that the problem (5)-(8) has a unique solution $(u, v)$ if $(u(R, \theta)$, $v(R, \theta))$ is given. This solution $(u, v)$ can be found in [13, §83]. For our application, the solution $(u, v)$ is given in the following form [7]:

$$
\begin{aligned}
& u(r, \theta)=\left(r^{2}-R^{2}\right) W_{1}+G_{1}, \\
& v(r, \theta)=\left(r^{2}-R^{2}\right) W_{2}+G_{2},
\end{aligned}
$$

where $x=r \cos \theta$ and $y=r \sin \theta$. Here $G_{1}, G_{2}, W_{1}$, and $W_{2}$ are harmonic functions, and

$$
\begin{aligned}
& G_{1}(r, \theta)=\frac{a_{0}}{2}+\sum_{n=1}^{\infty}\left(a_{n} \cos n \theta+b_{n} \sin n \theta\right) r^{-n} \\
& G_{2}(r, \theta)=\frac{c_{0}}{2}+\sum_{n=1}^{\infty}\left(c_{n} \cos n \theta+d_{n} \sin n \theta\right) r^{-n}
\end{aligned}
$$

with

$$
\begin{array}{ll}
a_{n}=\frac{R^{n}}{\pi} \int_{0}^{2 \pi} u(R, \theta) \cos n \theta d \theta, & n=0,1,2, \ldots, \\
b_{n}=\frac{R^{n}}{\pi} \int_{0}^{2 \pi} u(R, \theta) \sin n \theta d \theta, & n=1,2, \ldots, \\
c_{n}=\frac{R^{n}}{\pi} \int_{0}^{2 \pi} v(R, \theta) \cos n \theta d \theta, & n=0,1,2, \ldots, \\
d_{n}=\frac{R^{n}}{\pi} \int_{0}^{2 \pi} v(R, \theta) \sin n \theta d \theta, & n=1,2, \ldots
\end{array}
$$


Furthermore, let

$$
\frac{\partial u}{\partial x}+\frac{\partial v}{\partial y}=\Theta:=-\kappa p \quad \text { and } \quad \kappa=\frac{\mu}{\lambda+\mu} .
$$

Then we know that $p$ is a harmonic function, and

$$
p(r, \theta)=\sum_{n=2}^{\infty}\left(p_{n}^{1} \cos n \theta+p_{n}^{2} \sin n \theta\right) r^{-n},
$$

with

$$
\begin{aligned}
& \left(\frac{1}{2}+\kappa\right) p_{n}^{1}=(n-1)\left(a_{n-1}-d_{n-1}\right), \\
& \left(\frac{1}{2}+\kappa\right) p_{n}^{2}=(n-1)\left(b_{n-1}+c_{n-1}\right),
\end{aligned}
$$

and

$$
\begin{aligned}
& W_{1}(r, \theta)=\frac{1}{4} \sum_{n=2}^{\infty}\left\{p_{n}^{1} \cos (n+1) \theta+p_{n}^{2} \sin (n+1) \theta\right\} r^{-n-1}, \\
& W_{2}(r, \theta)=\frac{1}{4} \sum_{n=2}^{\infty}\left\{p_{n}^{1} \sin (n+1) \theta-p_{n}^{2} \cos (n+1) \theta\right\} r^{-n-1} .
\end{aligned}
$$

Finally, a computation shows

$$
\begin{gathered}
x W_{1}+y W_{2}=\frac{1}{4} p, \\
\left(\frac{1}{2}+\kappa\right) p=-\left(\frac{\partial G_{1}}{\partial x}+\frac{\partial G_{2}}{\partial y}\right) .
\end{gathered}
$$

We now discuss the stress on the boundary $\Gamma_{e}$. From

$$
X_{x}=\lambda \Theta+2 \mu \frac{\partial u}{\partial x}, \quad X_{y}=Y_{x}=\mu\left(\frac{\partial v}{\partial x}+\frac{\partial u}{\partial y}\right), \quad Y_{y}=\lambda \Theta+2 \mu \frac{\partial v}{\partial y},
$$

we obtain the vector components of stress acting on the boundary $\Gamma_{e}$ :

$$
X_{n}=\left.\left(X_{x} \cos \theta+X_{y} \sin \theta\right)\right|_{\Gamma_{e}}, \quad Y_{n}=\left.\left(X_{y} \cos \theta+Y_{y} \sin \theta\right)\right|_{\Gamma_{e}} .
$$

Furthermore, we get

$$
\begin{aligned}
X_{n}= & \left(\mu \frac{\partial u}{\partial r}-\lambda \kappa p \cos \theta+\mu \frac{\partial u}{\partial x} \cos \theta+\mu \frac{\partial v}{\partial x} \sin \theta\right)_{r=R} \\
= & \left\{\mu \frac{\partial u}{\partial r}-\lambda \kappa p \cos \theta+2 \mu x\left(W_{1} \cos \theta+W_{2} \sin \theta\right)\right. \\
& \left.+\mu \frac{\partial G_{1}}{\partial x} \cos \theta+\mu \frac{\partial G_{2}}{\partial x} \sin \theta\right\}_{r=R} \\
= & \mu\left(\frac{\partial u}{\partial r}-p \cos \theta+\frac{3 \mu+\lambda}{2(\lambda+\mu)} p \cos \theta+\frac{\partial G_{1}}{\partial x} \cos \theta+\frac{\partial G_{2}}{\partial x} \sin \theta\right)_{r=R} .
\end{aligned}
$$

The last equality comes from (17) and (23).

A computation shows

$$
\begin{aligned}
\left.\left(\frac{\partial u}{\partial r}-p \cos \theta\right)\right|_{r=R} & =2 R W_{1}(R, \theta)+\left.\frac{\partial G_{1}}{\partial r}\right|_{r=R}-p(R, \theta) \cos \theta \\
& =\left.\frac{2+2 \kappa}{1+2 \kappa} \frac{\partial G_{1}}{\partial r}\right|_{r=R}+\left.\frac{1}{(1+2 \kappa) R} \frac{\partial G_{2}}{\partial \theta}\right|_{r=R} .
\end{aligned}
$$


By equality (24) we have

$$
\begin{aligned}
\frac{3 \mu+\lambda}{2(\lambda+\mu)} p \cos \theta & =\frac{3 \mu+\lambda}{2(\lambda+\mu)} \frac{2}{1+2 \kappa}\left(-\left.\frac{\partial G_{1}}{\partial x}\right|_{r=R}-\left.\frac{\partial G_{2}}{\partial y}\right|_{r=R}\right) \cos \theta \\
& =-\left.\left(\frac{\partial G_{1}}{\partial x}+\frac{\partial G_{2}}{\partial y}\right)\right|_{r=R} \cos \theta .
\end{aligned}
$$

Hence, we get

$$
X_{n}(u, v)=\mu\left(\left.\frac{2+2 \kappa}{1+2 \kappa} \frac{\partial G_{1}}{\partial r}\right|_{r=R}-\left.\frac{2 \kappa}{(1+2 \kappa) R} \frac{\partial G_{2}}{\partial \theta}\right|_{r=R}\right)
$$

Similarly, we can get

$$
Y_{n}(u, v)=\mu\left(\left.\frac{2+2 \kappa}{1+2 \kappa} \frac{\partial G_{2}}{\partial r}\right|_{r=R}+\left|\frac{2 \kappa}{(1+2 \kappa) R} \frac{\partial G_{1}}{\partial \theta}\right|_{r=R}\right) .
$$

Substituting (11)-(16) into (25) and (26) and integrating by parts, we obtain the vector components of stress acting on the boundary $\Gamma_{e}$,

$$
\begin{aligned}
X_{n}(u, v) & =\frac{2+2 \kappa}{1+2 \kappa} \frac{\mu}{\pi R} \sum_{n=1}^{\infty} \int_{0}^{2 \pi} \frac{\partial^{2} u(R, \varphi)}{\partial \varphi^{2}} \frac{\cos n(\theta-\varphi)}{n} d \varphi \\
& -\frac{2 \kappa}{1+2 \kappa} \frac{\mu}{R} \frac{\partial v(R, \theta)}{\partial \theta} \\
& \equiv T_{1}(u, v), \\
Y_{n}(u, v) & =\frac{2+2 \kappa}{1+2 \kappa} \frac{\mu}{\pi R} \sum_{n=1}^{\infty} \int_{0}^{2 \pi} \frac{\frac{\partial^{2} v(R, \varphi)}{\partial \varphi^{2}} \frac{\cos n(\theta-\varphi)}{n} d \varphi}{+\frac{2 \kappa}{1+2 \kappa} \frac{\mu}{R} \frac{\partial u(R, \theta)}{\partial \theta}} \\
& \equiv T_{2}(u, v) .
\end{aligned}
$$

The formula can also be rewritten in the following form:

$$
\begin{aligned}
X_{n}(u, v)= & \frac{2+2 \kappa}{1+2 \kappa} \frac{\mu}{\pi R} \sum_{n=1}^{\infty} \int_{0}^{2 \pi} \frac{\partial^{2} u(R, \varphi)}{\partial \varphi^{2}} \frac{\cos n(\theta-\varphi)}{n} d \varphi \\
& -\frac{2 \kappa}{1+2 \kappa} \frac{\mu}{\pi R} \sum_{n=1}^{\infty} \int_{0}^{2 \pi} \frac{\partial^{2} v(R, \varphi)}{\partial \varphi^{2}} \frac{\sin n(\theta-\varphi)}{n} d \varphi \\
Y_{n}(u, v)= & \frac{2+2 \kappa}{1+2 \kappa} \frac{\mu}{\pi R} \sum_{n=1}^{\infty} \int_{0}^{2 \pi} \frac{\partial^{2} v(R, \varphi)}{\partial \varphi^{2}} \frac{\cos n(\theta-\varphi)}{n} d \varphi \\
& +\frac{2 \kappa}{1+2 \kappa} \frac{\mu}{\pi R} \sum_{n=1}^{\infty} \int_{0}^{2 \pi} \frac{\partial^{2} u(R, \varphi)}{\partial \varphi^{2}} \frac{\sin n(\theta-\varphi)}{n} d \varphi .
\end{aligned}
$$

We now get the exact boundary condition $(27)-(28)$ (or $(29)-(30)$ ) at the artificial boundary $\Gamma_{e}$. Then the restriction of the solution $(u, v)$ of problem 
(1)-(4) to the bounded domain $\Omega_{i}$ is a solution of the following problem:

$$
\begin{aligned}
-\mu \Delta u-(\lambda+\mu) \frac{\partial}{\partial x}\left(\frac{\partial u}{\partial x}+\frac{\partial v}{\partial y}\right) & =f_{1} \text { in } \Omega_{i}, \\
-\mu \Delta v-(\lambda+\mu) \frac{\partial}{\partial y}\left(\frac{\partial u}{\partial x}+\frac{\partial v}{\partial y}\right) & =f_{2} \text { in } \Omega_{i}, \\
u & =0 \text { on } \Gamma_{i}, \\
v & =0 \text { on } \Gamma_{i}, \\
X_{n} & =T_{1}(u, v) \text { on } \Gamma_{e}, \\
Y_{n} & =T_{2}(u, v) \text { on } \Gamma_{e} .
\end{aligned}
$$

This is a boundary value problem with global boundary condition on $\Gamma_{e}$. Let

$$
\begin{aligned}
T_{1}^{N}(u, v)= & \frac{2+2 \kappa}{1+2 \kappa} \frac{\mu}{\pi R} \sum_{n=1}^{N} \int_{0}^{2 \pi} \frac{\partial^{2} u(R, \varphi)}{\partial \varphi^{2}} \frac{\cos n(\theta-\varphi)}{n} d \varphi \\
& -\frac{2 \kappa}{1+2 \kappa} \frac{\mu}{\pi R} \sum_{n=1}^{N} \int_{0}^{2 \pi} \frac{\partial^{2} v(R, \varphi)}{\partial \varphi^{2}} \frac{\sin n(\theta-\varphi)}{n} d \varphi \\
T_{2}^{N}(u, v)= & \frac{2+2 \kappa}{1+2 \kappa} \frac{\mu}{\pi R} \sum_{n=1}^{N} \int_{0}^{2 \pi} \frac{\partial^{2} v(R, \varphi)}{\partial \varphi^{2}} \frac{\cos n(\theta-\varphi)}{n} d \varphi \\
& +\frac{2 \kappa}{1+2 \kappa} \frac{\mu}{\pi R} \sum_{n=1}^{N} \int_{0}^{2 \pi} \frac{\partial^{2} u(R, \varphi)}{\partial \varphi^{2}} \frac{\sin n(\theta-\varphi)}{n} d \varphi
\end{aligned}
$$

and $T_{1}^{0}(u, v)=0, T_{2}^{0}(u, v)=0$. Then we get a sequence of approximate boundary condition on the artificial boundary $\Gamma_{e}$,

$$
\begin{aligned}
& X_{n}=T_{1}^{N}(u, v) \text { on } \Gamma_{e}, \\
& Y_{n}=T_{2}^{N}(u, v) \text { on } \Gamma_{e} \text {, }
\end{aligned}
$$

for $N=0,1,2, \ldots$. When $N=0$, then (37)-(38) reduces to

$$
X_{n}=0 \text { on } \Gamma_{e}, \quad Y_{n}=0 \text { on } \Gamma_{e},
$$

which is often used in engineering.

By means of the approximate boundary condition (37)-(38), we reduce the original problem (1)-(4) to the following problem on the bounded domain $\Omega_{i}$ approximately for $N=0,1,2, \ldots$ :

$$
\begin{aligned}
-\mu \Delta u-(\lambda+\mu) \frac{\partial}{\partial x}\left(\frac{\partial u}{\partial x}+\frac{\partial v}{\partial y}\right) & =f_{1} \text { in } \Omega_{i}, \\
-\mu \Delta v-(\lambda+\mu) \frac{\partial}{\partial y}\left(\frac{\partial u}{\partial x}+\frac{\partial v}{\partial y}\right) & =f_{2} \text { in } \Omega_{i}, \\
u & =0 \text { on } \Gamma_{i}, \\
v & =0 \text { on } \Gamma_{i}, \\
X_{n} & =T_{1}^{N}(u, v) \text { on } \Gamma_{e}, \\
Y_{n} & =T_{2}^{N}(u, v) \text { on } \Gamma_{e} .
\end{aligned}
$$


In the following section we will show that the boundary value problems (31)(36) and (39)-(44) are well posed.

3. The SOlution OF PROBlems (31)-(36) AND (39)-(44)

Let $H^{m}\left(\Omega_{i}\right)$ and $H^{s}\left(\Gamma_{e}\right)$ denote the usual Sobolev spaces on the domain $\Omega_{i}$ and the boundary $\Gamma_{e}$, with integer $m$ and real $s$. Furthermore, let

$$
\begin{gathered}
H_{*}^{1}\left(\Omega_{i}\right)=\left\{v \in H^{1}\left(\Omega_{i}\right) ;\left.v\right|_{\Gamma_{i}}=0\right\} \quad \text { with norm }\|v\|_{1, \Omega_{i}}, \\
V=H_{*}^{1}\left(\Omega_{i}\right) \times H_{*}^{1}\left(\Omega_{i}\right) \quad \text { with norm }\|(u, v)\|_{V}^{2}=\|u\|_{1, \Omega_{i}}^{2}+\|v\|_{1, \Omega_{i}}^{2} .
\end{gathered}
$$

Then the boundary value problem $(31)-(36)$ is equivalent to the following variational problem:

Find $(u, v) \in V$ such that

$$
\begin{aligned}
& A(u, v ; \tilde{u}, \tilde{v})+B(u, v ; \tilde{u}, \tilde{v}) \\
& \quad=\iint_{\Omega_{i}}\left(f_{1} \tilde{u}+f_{2} \tilde{v}\right) d x d y \quad \forall(\tilde{u}, \tilde{v}) \in V
\end{aligned}
$$

where

$$
\begin{array}{r}
A(u, v ; \tilde{u}, \tilde{v}) \\
=\iint_{\Omega_{i}}\left\{\lambda\left(\frac{\partial u}{\partial x}+\frac{\partial v}{\partial y}\right)\left(\frac{\partial \tilde{u}}{\partial x}+\frac{\partial \tilde{v}}{\partial y}\right)+2 \mu\left(\frac{\partial u}{\partial x} \frac{\partial \tilde{u}}{\partial x}+\frac{\partial v}{\partial y} \frac{\partial \tilde{v}}{\partial y}\right)\right. \\
\left.+\mu\left(\frac{\partial v}{\partial x}+\frac{\partial u}{\partial y}\right)\left(\frac{\partial \tilde{v}}{\partial x}+\frac{\partial \tilde{u}}{\partial y}\right)\right\} d x d y \\
\forall(u, v),(\tilde{u}, \tilde{v}) \in V, \\
B(u, v ; \tilde{u}, \tilde{v}) \\
=\frac{2+2 \kappa}{1+2 \kappa} \frac{\mu}{\pi} \sum_{n=1}^{\infty} \int_{0}^{2 \pi} \int_{0}^{2 \pi}\left\{\frac{\partial u(R, \varphi)}{\partial \varphi} \frac{\partial \tilde{u}(R, \theta)}{\partial \theta}+\frac{\partial v(R, \varphi)}{\partial \varphi} \frac{\partial \tilde{v}(R, \theta)}{\partial \theta}\right\} \\
+\frac{2 \kappa}{1+2 \kappa} \frac{\mu}{\pi} \sum_{n=1}^{\infty} \int_{0}^{2 \pi} \int_{0}^{2 \pi}\left\{\begin{array}{c}
\left.-\frac{\partial v(R, \varphi)}{\partial \varphi} \frac{\partial \tilde{u}(R, \theta)}{\partial \theta}+\frac{\partial u(R, \varphi)}{\partial \varphi} \frac{\partial \tilde{v}(R, \theta)}{\partial \theta}\right\} \\
\cdot \frac{\sin n(\theta-\varphi)}{n} d \varphi d \theta \quad \forall(u, v),(\tilde{u}, \tilde{v}) \in V .
\end{array}\right.
\end{array}
$$

Furthermore, let

$$
\begin{array}{r}
B_{N}(u, v ; \tilde{u}, \tilde{v}) \\
=\frac{2+2 \kappa}{1+2 \kappa} \frac{\mu}{\pi} \sum_{n=1}^{N} \int_{0}^{2 \pi} \int_{0}^{2 \pi}\left\{\frac{\partial u(R, \varphi)}{\partial \varphi} \frac{\partial \tilde{u}(R, \theta)}{\partial \theta}+\frac{\partial v(R, \varphi)}{\partial \varphi} \frac{\partial \tilde{v}(R, \theta)}{\partial \theta}\right\} \\
+\frac{2 \kappa}{1+2 \kappa} \frac{\mu}{\pi} \sum_{n=1}^{N} \int_{0}^{2 \pi} \int_{0}^{2 \pi}\left\{-\frac{\cos n(\theta-\varphi)}{n} d \varphi d \theta\right. \\
\cdot \frac{\sin n(\theta-\varphi)}{n} d \varphi d \theta \quad \forall(u, v),(\tilde{u}, \tilde{v}) \in V .
\end{array}
$$


Then the boundary value problem (39)-(44) is equivalent to the following variational problem:

$$
\begin{aligned}
& \text { Find }\left(u_{N}, v_{N}\right) \in V \text { such that } \\
& \qquad \begin{aligned}
A\left(u_{N}, v_{N} ; \tilde{u}, \tilde{v}\right)+B_{N}\left(u_{N}, v_{N} ; \tilde{u}, \tilde{v}\right) \\
=\iint_{\Omega_{i}}\left(f_{1} \tilde{u}+f_{2} \tilde{v}\right) d x d y \quad \forall(\tilde{u}, \tilde{v}) \in V .
\end{aligned}
\end{aligned}
$$

From Korn's inequality [12], we know that the following holds.

Lemma 1. The bilinear form $A(u, v ; \tilde{u}, \tilde{v})$ is symmetric, bounded, and coercive on $V \times V$. That is, there are two positive constants $M_{0}$ and $\beta_{0}$ such that

$$
\begin{gathered}
|A(u, v ; \tilde{u}, \tilde{v})| \leq M_{0}\|(u, v)\|_{V}\|(\tilde{u}, \tilde{v})\|_{V} \quad \forall(u, v),(\tilde{u}, \tilde{v}) \in V, \\
A(u, v ; u, v) \geq \beta_{0}\|(u, v)\|_{V}^{2} \quad \forall(u, v) \in V .
\end{gathered}
$$

For the bilinear forms $B(u, v ; \tilde{u}, \tilde{v})$ and $B_{N}(u, v ; \tilde{u}, \tilde{v})$, we have

Lemma 2. The bilinear forms $B(u, v ; \tilde{u}, \tilde{v})$ and $B_{N}(u, v ; \tilde{u}, \tilde{v})$ are symmetric and bounded on $V \times V$, i.e., there is a constant $M_{1} \geq 0$ such that

$$
\begin{aligned}
|B(u, v ; \tilde{u}, \tilde{v})| \leq M_{1}\|(u, v)\|_{V}\|(\tilde{u}, \tilde{v})\|_{V} & \forall(u, v),(\tilde{u}, \tilde{v}) \in V, \\
\left|B_{N}(u, v ; \tilde{u}, \tilde{v})\right| \leq M_{1}\|(u, v)\|_{V}\|(\tilde{u}, \tilde{v})\|_{V} & \forall(u, v),(\tilde{u}, \tilde{v}) \in V .
\end{aligned}
$$

Furthermore,

$$
\begin{aligned}
B(u, v ; u, v) \geq 0 & \forall(u, v) \in V, \\
B_{N}(u, v ; u, v) \geq 0 & \forall(u, v) \in V .
\end{aligned}
$$

Proof. We recall an equivalent definition of Sobolev space $H^{s}\left(\Gamma_{e}\right)$ [14]:

$$
\begin{aligned}
& u \in H^{s}\left(\Gamma_{e}\right) \Leftrightarrow u=\frac{a_{0}}{2}+\sum_{n=1}^{\infty}\left(a_{n} \cos n \theta+b_{n} \sin n \theta\right) \\
& \text { and }\|u\|_{\Delta, s}=\left(\frac{a_{0}}{2}+\sum_{n=1}^{\infty}\left(1+n^{2}\right)^{s}\left(a_{n}^{2}+b_{n}^{2}\right)\right)^{1 / 2} \leq \infty,
\end{aligned}
$$

where $\|u\|_{\Delta, s}$ is an equivalent norm in $H^{s}\left(\Gamma_{e}\right)$.

For any $(u, v),(\tilde{u}, \tilde{v}) \in V$ we know that $\left.u\right|_{\Gamma_{e}},\left.v\right|_{\Gamma_{e}},\left.\tilde{u}\right|_{\Gamma_{e}}$, and $\left.\tilde{v}\right|_{\Gamma_{e}}$ belong to the space $H^{1 / 2}\left(\Gamma_{e}\right)$ by the trace theorem. Assume

$$
\begin{aligned}
& u(R, \theta)=\frac{a_{0}}{2}+\sum_{n=1}^{\infty}\left(a_{n} \cos n \theta+b_{n} \sin n \theta\right), \\
& v(R, \theta)=\frac{c_{0}}{2}+\sum_{n=1}^{\infty}\left(c_{n} \cos n \theta+d_{n} \sin n \theta\right), \\
& \tilde{u}(R, \theta)=\frac{\tilde{a}_{0}}{2}+\sum_{n=1}^{\infty}\left(\tilde{a}_{n} \cos n \theta+\tilde{b}_{n} \sin n \theta\right), \\
& \tilde{v}(R, \theta)=\frac{\tilde{c}_{0}}{2}+\sum_{n=1}^{\infty}\left(\tilde{c}_{n} \cos n \theta+\tilde{d}_{n} \sin n \theta\right) .
\end{aligned}
$$


Then

$$
\begin{aligned}
\|u(R, \theta)\|_{\Delta, 1 / 2} & =\left(\frac{a_{0}}{2}+\sum_{n=1}^{\infty}\left(1+n^{2}\right)^{1 / 2}\left(a_{n}^{2}+b_{n}^{2}\right)\right)^{1 / 2}<\infty, \\
\|v(R, \theta)\|_{\Delta, 1 / 2} & =\left(\frac{c_{0}}{2}+\sum_{n=1}^{\infty}\left(1+n^{2}\right)^{1 / 2}\left(c_{n}^{2}+d_{n}^{2}\right)\right)^{1 / 2}<\infty, \\
\|\tilde{u}(R, \theta)\|_{\Delta, 1 / 2} & =\left(\frac{\tilde{a}_{0}}{2}+\sum_{n=1}^{\infty}\left(1+n^{2}\right)^{1 / 2}\left(\tilde{a}_{n}^{2}+\tilde{b}_{n}^{2}\right)\right)^{1 / 2}<\infty, \\
\|\tilde{v}(R, \theta)\|_{\Delta, 1 / 2} & =\left(\frac{\tilde{c}_{0}}{2}+\sum_{n=1}^{\infty}\left(1+n^{2}\right)^{1 / 2}\left(\tilde{c}_{n}^{2}+\tilde{d}_{n}^{2}\right)\right)^{1 / 2}<\infty
\end{aligned}
$$

A computation shows

$$
\begin{aligned}
B(u, v ; \tilde{u}, \tilde{v})= & \frac{2(1+\kappa) \pi \mu}{(1+2 \kappa)} \sum_{n=1}^{\infty} n\left\{a_{n} \tilde{a}_{n}+b_{n} \tilde{b}_{n}+c_{n} \tilde{c}_{n}+d_{n} \tilde{d}_{n}\right\} \\
& +\frac{2 \kappa \pi \mu}{(1+2 \kappa)} \sum_{n=1}^{\infty} n\left\{a_{n} \tilde{d}_{n}-b_{n} \tilde{c}_{n}-c_{n} \tilde{b}_{n}+d_{n} \tilde{a}_{n}\right\} \\
= & \frac{2 \pi \mu}{(1+2 \kappa)} \sum_{n=1}^{\infty} n\left\{(1+\kappa)\left(a_{n} \tilde{a}_{n}+b_{n} \tilde{b}_{n}+c_{n} \tilde{c}_{n}+d_{n} \tilde{d}_{n}\right)\right. \\
\leq & \frac{4(1+\kappa) \pi \mu}{(1+2 \kappa)}\left\{\sum_{n=1}^{\infty} n\left(a_{n}^{2} \tilde{d}_{n}-b_{n} \tilde{c}_{n}-c_{n} \tilde{b}_{n}+d_{n} \tilde{a}_{n}\right)\right\} \\
& \left.\cdot\left\{\sum_{n}^{2}+d_{n}^{2}\right)\right\}^{1 / 2} \\
\leq & \frac{4(1+\kappa) \pi \mu}{(1+2 \kappa)}\|(u, v)\|_{\Delta, 1 / 2}\|(\tilde{u}, \tilde{v})\|_{\Delta, 1 / 2} \\
\leq & \frac{4(1+\kappa) \pi \mu c}{(1+2 \kappa)}\|(u, v)\|_{1 / 2}^{2}+\tilde{c}_{e}^{2}\|(\tilde{u}, \tilde{v})\|_{1 / 2}^{2}, \Gamma_{e},
\end{aligned}
$$

where the last inequality is a consequence of the fact that $\|u\|_{\Delta, 1 / 2}$ is equivalent to the norm $\|u\|_{1 / 2, \Gamma_{e}}$ in the Sobolev space $H^{1 / 2}\left(\Gamma_{e}\right)$, and $c$ is a constant independent of $N$. By the trace theorem we obtain the inequality (47).

Furthermore,

$$
\begin{aligned}
B(u, v ; u, v)=\frac{2 \pi \mu}{(1+2 \kappa)} \sum_{n=1}^{\infty} n & \left\{a_{n}^{2}+b_{n}^{2}+c_{n}^{2}+d_{n}^{2}\right. \\
& \left.+\kappa\left(b_{n}-c_{n}\right)^{2}+\kappa\left(a_{n}+d_{n}\right)^{2}\right\} \geq 0 \quad \forall(u, v) \in V .
\end{aligned}
$$

Similarly, for $B_{N}(u, v ; \tilde{u}, \tilde{v})$ we obtain

$$
\begin{aligned}
\left|B_{N}(u, v ; \tilde{u}, \tilde{v})\right| & \leq M_{1}\|(u, v)\|_{V}\|(\tilde{u}, \tilde{v})\|_{V} \quad \forall(u, v),(\tilde{u}, \tilde{v}) \in V, \\
B_{N}(u, v ; u, v) & \geq 0 \quad \forall(u, v) \in V .
\end{aligned}
$$

The proof of Lemma 2 is completed. 
On the other hand, we have

$$
\begin{aligned}
&\left|B(u, v ; \tilde{u}, \tilde{v})-B_{N}(u, v ; \tilde{u}, \tilde{v})\right| \\
&=\mid \frac{2(1+\kappa) \pi \mu}{(1+2 \kappa)} \sum_{n=N+1}^{\infty} n\left\{a_{n} \tilde{a}_{n}+b_{n} \tilde{b}_{n}+c_{n} \tilde{c}_{n}+d_{n} \tilde{d}_{n}\right\} \\
& \quad+\frac{2 \kappa \pi \mu}{(1+2 \kappa)} \sum_{n=N+1}^{\infty} n\left\{a_{n} \tilde{d}_{n}-b_{n} \tilde{c}_{n}-c_{n} \tilde{b}_{n}+d_{n} \tilde{a}_{n}\right\} \mid \\
& \leq \frac{4(1+\kappa) \pi \mu}{(1+2 \kappa)}\left\{\sum_{n=N+1}^{\infty} n\left(a_{n}^{2}+b_{n}^{2}+c_{n}^{2}+d_{n}^{2}\right)\right\}^{1 / 2} \\
& \cdot\left\{\sum_{n=N+1}^{\infty} n\left(\tilde{a}_{n}^{2}+\tilde{b}_{n}^{2}+\tilde{c}_{n}^{2}+\tilde{d}_{n}^{2}\right)\right\}^{1 / 2} \\
& \leq \frac{4(1+\kappa) \pi \mu}{(1+2 \kappa) N^{k-1}\left\{\sum_{n=N+1}^{\infty}\left(n^{2}\right)^{k-1 / 2}\left(a_{n}^{2}+b_{n}^{2}+c_{n}^{2}+d_{n}^{2}\right)\right\}^{1 / 2}} \\
& \cdot\left\{\sum_{n=N+1}^{\infty} n\left(\tilde{a}_{n}^{2}+\tilde{b}_{n}^{2}+\tilde{c}_{n}^{2}+\tilde{d}_{n}^{2}\right)\right\}^{1 / 2} \\
& \leq \frac{c}{N^{k-1}}\|(u, v)\|_{k-1 / 2, \Gamma_{e}}\|(\tilde{u}, \tilde{v})\|_{1 / 2, \Gamma_{e}}, \quad \forall k \geq 2 .
\end{aligned}
$$

Hence we obtain the following error estimate:

Lemma 3. The following error estimate holds:

$$
\left|B(u, v ; \tilde{u}, \tilde{v})-B_{N}(u, v ; \tilde{u}, \tilde{v})\right| \leq \frac{c}{N^{k-1}}\|(u, v)\|_{k-1 / 2, \Gamma_{e}}\|(\tilde{u}, \tilde{v})\|_{1 / 2, \Gamma_{e}},
$$

with $k \geq 2$ and $c$ a constant independent of $N,(u, v)$, and $(\tilde{u}, \tilde{v})$.

Theorem 1. Suppose $f_{1}, f_{2} \in H^{-1}\left(\Omega_{i}\right)$; then the variational problem (45) has a unique solution $(u, v) \in V$ and problem (46) has a unique solution $\left(u_{N}, v_{N}\right) \in$ $V$. Furthermore, we have the following error estimate:

$$
\left\|\left(u-u_{N}, v-v_{N}\right)\right\|_{V} \leq \frac{c}{\beta_{0} N^{k-1}}\|(u, v)\|_{k-1 / 2, \Gamma_{e}}
$$

Proof. By Lemmas 1 and 2, we know that $A(u, v ; \tilde{u}, \tilde{v})+B(u, v ; \tilde{u}, \tilde{v})$ and $A(u, v ; \tilde{u}, \tilde{v})+B_{N}(u, v ; \tilde{u}, \tilde{v})$ are two symmetric, bounded, and coercive bilinear functionals on $V \times V$. By Cauchy's inequality, $\left(f_{1}, f_{2} ; \tilde{u}, \tilde{v}\right)$ is a linear functional on $V$. From the Lax-Milgram theorem [2], we obtain that the problem (45) has a unique solution $(u, v)$ which is the restriction to $\Omega_{i}$ of the solution $(u, v)$ of the original problem (1)-(4), and the problem (46) has a unique solution $\left(u_{N}, v_{N}\right)$.

Let $e_{1}=u-u_{N}$ and $e_{2}=v-v_{N}$; then $\left(e_{1}, e_{2}\right)$ satisfies

$$
\begin{aligned}
& A\left(e_{1}, e_{2} ; \tilde{u}, \tilde{v}\right)+B_{N}\left(e_{1}, e_{2} ; \tilde{u}, \tilde{v}\right) \\
& \quad=B_{N}(u, v ; \tilde{u}, \tilde{v})-B(u, v ; \tilde{u}, \tilde{v}) \quad \forall(\tilde{u}, \tilde{v}) \in V .
\end{aligned}
$$


Taking $\tilde{u}=e_{1}$ and $\tilde{v}=e_{2}$ in (51), we get

$$
\begin{aligned}
\beta_{0}\left\|\left(e_{1}, e_{1}\right)\right\|_{V}^{2} & \leq A\left(e_{1}, e_{2} ; e_{1}, e_{2}\right) \leq\left|B_{N}\left(u, v ; e_{1}, e_{2}\right)-B\left(u, v ; e_{1}, e_{2}\right)\right| \\
& \leq \frac{c}{N^{k-1}}\|(u, v)\|_{k-1 / 2, \Gamma_{e}}\left\|\left(e_{1}, e_{2}\right)\right\|_{V} .
\end{aligned}
$$

The last inequality comes from Lemma 3. The inequality (50) now follows immediately.

\section{The FINITE ELEMENT APPROXIMATION OF PROBLEM (46)}

For the sake of simplicity, let $\Gamma_{i}$ be a polygonal line, and $\mathscr{T}_{h}$ be a triangulation of $\Omega_{i}$ satisfying

$$
\Omega_{i}=\left(\bigcup_{K \in \mathscr{T}_{h}} K\right) \cap\left(\bigcup_{\tilde{K} \in \mathscr{T}_{h}} \tilde{K}\right),
$$

where $K$ is a triangle and $\widetilde{K}$ is a curved triangle with a curved side on $\Gamma_{e}$, and

$$
\frac{h_{k}}{\rho_{k}} \leq \sigma \quad \forall K, \widetilde{K} \in \mathscr{T}_{h},
$$

where $h_{k}=$ diameter of $K$ or $\tilde{K}, \rho_{k}=$ diameter of the inscribed circle of $K$ or $\tilde{K}$, and $h=\max _{K, \widetilde{K} \in \mathscr{T}_{h}} h_{k}$. Let

$$
\begin{gathered}
S_{h}\left(\Omega_{i}\right)=\left\{v \in H_{*}^{1}\left(\Omega_{i}\right),\left.\quad v\right|_{K}\left(\left.v\right|_{\tilde{K}}\right) \text { is a linear polynomial } \forall K(\tilde{K}) \in \mathscr{T}_{h}\right\}, \\
V_{h}=S_{h}\left(\Omega_{i}\right) \times S_{h}\left(\Omega_{i}\right) .
\end{gathered}
$$

We know that the subspace $V_{h}$ is a regular finite element space in the sense of Babuška and Aziz [1], which satisfies the following approximation property:

$$
\inf _{\left(u_{h}, v_{h}\right) \in V_{h}}\left\|\left(u-u_{h}, v-v_{h}\right)\right\|_{V} \leq c h\|(u, v)\|_{2, \Omega_{i}} \text {. }
$$

We now consider the approximation problem of (46):

$$
\text { Find }\left(u_{N}^{h}, v_{N}^{h}\right) \in V_{h} \text { such that }
$$

$$
\begin{aligned}
& A\left(u_{N}^{h}, v_{N}^{h} ; \tilde{u}, \tilde{v}\right)+B_{N}\left(u_{N}^{h}, v_{N}^{h} ; \tilde{u}, \tilde{v}\right) \\
& \quad=\iint_{\Omega_{i}}\left(f_{1} \tilde{u}+f_{2} \tilde{v}\right) d x d y \quad \forall(\tilde{u}, \tilde{v}) \in V_{h} .
\end{aligned}
$$

By the Lax-Milgram theorem we have

Theorem 2. The variational problem (53) has a unique solution $\left(u_{N}^{h}, v_{N}^{h}\right) \in V_{h}$.

Theorem 3. Assume that $u, v \in H^{2}\left(\Omega_{i}\right) \cap H^{k-1 / 2}\left(\Gamma_{e}\right), k \geq 2$, where $(u, v)$ is the solution of problem (45); then the following error estimate holds:

$$
\left\|\left(u-u_{N}^{h}, v-v_{N}^{h}\right)\right\|_{V} \leq c\left\{h\|(u, v)\|_{2, \Omega_{i}}+\frac{1}{N^{k-1}}\|(u, v)\|_{k-1 / 2, \Gamma_{e}}\right\},
$$

where $c$ is a constant independent of $h$ and $N$.

Proof. From the equalities (45) and (53) we obtain

$$
\begin{array}{r}
A\left(u-u_{N}^{h}, v-v_{N}^{h} ; \tilde{u}, \tilde{v}\right)+B_{N}\left(u-u_{N}^{h}, v-v_{N}^{h} ; \tilde{u}, \tilde{v}\right) \\
\quad=B_{N}(u, v ; \tilde{u}, \tilde{v})-B(u, v ; \tilde{u}, \tilde{v}) \quad \forall(\tilde{u}, \tilde{v}) \in V_{h} .
\end{array}
$$


Then

$$
\begin{aligned}
& \left\|\left(u_{N}^{h}-\tilde{u}, v_{N}^{h}-\tilde{v}\right)\right\|_{V}^{2} \\
& \leq \frac{1}{\beta_{0}}\left\{A\left(u_{N}^{h}-\tilde{u}, v_{N}^{h}-\tilde{v} ; u_{N}^{h}-\tilde{u}, v_{N}^{h}-\tilde{v}\right)\right. \\
& \left.+B_{N}\left(u_{N}^{h}-\tilde{u}, v_{N}^{h}-\tilde{v} ; u_{N}^{h}-\tilde{u}, v_{N}^{h}-\tilde{v}\right)\right\} \\
& =\frac{1}{\beta_{0}}\left\{A\left(u-\tilde{u}, v-\tilde{v} ; u_{N}^{h}-\tilde{u}, v_{N}^{h}-\tilde{v}\right)\right. \\
& +B_{N}\left(u-\tilde{u}, v-\tilde{v} ; u_{N}^{h}-\tilde{u}, v_{N}^{h}-\tilde{v}\right) \\
& \left.+B\left(u, v ; u_{N}^{h}-\tilde{u}, v_{N}^{h}-\tilde{v}\right)-B_{N}\left(u, v ; u_{N}^{h}-\tilde{u}, v_{N}^{h}-\tilde{v}\right)\right\} \\
& \leq \frac{\left(M_{0}+M_{1}\right)}{\beta_{0}}\|(u-\tilde{u}, v-\tilde{v})\|_{V}\left\|\left(u_{N}^{h}-\tilde{u}, v_{N}^{h}-\tilde{v}\right)\right\|_{V} \\
& +\frac{c}{\beta_{0} N^{k-1}}\|(u, v)\|_{k-1 / 2, \Gamma_{e}}\left\|\left(u_{N}^{h}-\tilde{u}, v_{N}^{h}-\tilde{v}\right)\right\|_{V} \quad \forall(\tilde{u}, \tilde{v}) \in V_{h} .
\end{aligned}
$$

Therefore, we have

$$
\begin{aligned}
\left\|\left(u_{N}^{h}-\tilde{u}, v_{N}^{h}-\tilde{v}\right)\right\|_{V} \leq & \frac{\left(M_{0}+M_{1}\right)}{\beta_{0}}\|(u-\tilde{u}, v-\tilde{v})\|_{V} \\
& +\frac{c}{\beta_{0} N^{k-1}}\|(u, v)\|_{k-1 / 2, \Gamma_{e}} \quad \forall(\tilde{u}, \tilde{v}) \in V_{h} .
\end{aligned}
$$

By the triangle inequality,

$$
\begin{aligned}
\left\|\left(u-u_{N}^{h}, v-v_{N}^{h}\right)\right\|_{V} \leq & \|(u-\tilde{u}, v-\tilde{v})\|_{V}+\left\|\left(u_{N}^{h}-\tilde{u}, v_{N}^{h}-\tilde{v}\right)\right\|_{V} \\
\leq & \left(\frac{\left(M_{0}+M_{1}\right)}{\beta_{0}}+1\right)\|(u-\tilde{u}, v-\tilde{v})\|_{V} \\
& +\frac{c}{\beta_{0} N^{k-1}}\|(u, v)\|_{k-1 / 2, \Gamma_{e}}
\end{aligned}
$$

Hence, we get

$$
\begin{aligned}
\left\|\left(u-u_{N}^{h}, v-v_{N}^{h}\right)\right\|_{V} \leq & \frac{M_{0}+M_{1}+\beta_{0}}{\beta_{0}} \inf _{(\tilde{u}, \tilde{v}) \in V_{h}}\|(u-\tilde{u}, v-\tilde{v})\|_{V} \\
& +\frac{c}{\beta_{0} N^{k-1}}\|(u, v)\|_{k-1 / 2, \Gamma_{e}} .
\end{aligned}
$$

By inequality (52), the proof is completed.

\section{NUMERICAL EXAMPLE}

Suppose that the unbounded domain $\Omega=\{(x, y) \in \Omega, 1<|x|$ or $1<|y|\}$ is the exterior domain of the square $[-1,1] \times[-1,1]$ with boundary $\Gamma_{i}$. Let

$$
\begin{aligned}
& u_{1}(x, y)=\frac{\lambda+3 \mu}{4 \pi \mu(\lambda+2 \mu)}\{ \frac{1}{2} \log \frac{x^{2}+(y+0.5)^{2}}{x^{2}+(y-0.5)^{2}} \\
&\left.+\frac{\lambda+\mu}{\lambda+3 \mu}\left(\frac{x^{2}}{x^{2}+(y-0.5)^{2}}-\frac{x^{2}}{x^{2}+(y+0.5)^{2}}\right)\right\}, \\
& v_{1}(x, y)=\frac{\lambda+\mu}{4 \pi \mu(\lambda+2 \mu)}\left(\frac{x(y-0.5)}{x^{2}+(y-0.5)^{2}}-\frac{x(y+0.5)}{x^{2}+(y+0.5)^{2}}\right) .
\end{aligned}
$$






FIGURE 2

Then $\left(u_{1}, v_{1}\right)$ is the unique solution of the following boundary value problem:

$$
\begin{aligned}
-\mu \Delta u-(\lambda+\mu) \frac{\partial}{\partial x}\left(\frac{\partial u}{\partial x}+\frac{\partial v}{\partial y}\right) & =0 & & \text { in } \Omega, \\
-\mu \Delta v-(\lambda+\mu) \frac{\partial}{\partial y}\left(\frac{\partial u}{\partial x}+\frac{\partial v}{\partial y}\right) & =0 & & \text { in } \Omega, \\
u & =u_{1} & & \text { on } \Gamma_{i}, \\
v & =v_{1} & & \text { on } \Gamma_{i},
\end{aligned}
$$

$u, v$ are bounded when $r \rightarrow+\infty$.

We take $\Gamma_{e}$ as a circumference with radius 2 ; then we consider the finite element approximation of $(u, v)$ on the bounded domain $\Omega_{i}=\{(x, y) \in \Omega$ and $r<2\}$.

Since $u_{1}$ and $v_{1}$ are symmetric about the $x$ and $y$ axes, respectively, and antisymmetric about the $y$ and $x$ axes, respectively, the domain of computation was taken to be the part lying in the first quadrant. The symmetric and antisymmetric boundary conditions were used along $x=0$ and $y=0$.

Three meshes were used in computation. Figure 2 shows the triangulation for mesh A. Mesh B was generated by dividing the triangles in mesh A into four small triangles, and mesh $\mathrm{C}$ was similarly generated. Linear finite element approximation was used in computation. Table 1 shows the maximum of the errors $u-u_{N}^{h}$ and $v-v_{N}^{h}$ over the mesh points when $N=5$. Since the maximum norm of $u$ is about 0.117 , the maximum relative error for $u$ is

TABLE 1. Maximum error for $N=5$

\begin{tabular}{|c|c|c|c|}
\hline mesh & A & B & C \\
\hline$h$ & 0.36 & 0.18 & 0.09 \\
\hline $\max \left|u_{i}-u_{N, i}^{h}\right|$ & $0.370 \mathrm{~d}-02$ & $0.117 \mathrm{~d}-02$ & $0.294 \mathrm{~d}-03$ \\
\hline $\max \left|v_{i}-v_{N, i}^{h}\right|$ & $0.651 \mathrm{~d}-02$ & $0.252 \mathrm{~d}-02$ & $0.840 \mathrm{~d}-03$ \\
\hline
\end{tabular}


TABle 2. Maximum error for mesh A

\begin{tabular}{|c|c|c|c|c|}
\hline$N$ & 0 & 1 & 3 & 5 \\
\hline $\max \left|u_{i}-u_{N, i}^{h}\right|$ & $0.433 \mathrm{~d}-01$ & $0.610 \mathrm{~d}-02$ & $0.358 \mathrm{~d}-02$ & $0.370 \mathrm{~d}-02$ \\
\hline $\max \left|v_{i}-v_{N, i}^{h}\right|$ & $0.721 \mathrm{~d}-02$ & $0.148 \mathrm{~d}-01$ & $0.658 \mathrm{~d}-02$ & $0.651 \mathrm{~d}-02$ \\
\hline
\end{tabular}

TABLE 3. Maximum error for mesh B

\begin{tabular}{|c|c|c|c|c|}
\hline$N$ & 0 & 1 & 3 & 5 \\
\hline $\max \left|u_{i}-u_{N, i}^{h}\right|$ & $0.412 \mathrm{~d}-01$ & $0.521 \mathrm{~d}-02$ & $0.116 \mathrm{~d}-02$ & $0.117 \mathrm{~d}-02$ \\
\hline $\max \left|v_{i}-v_{N, i}^{h}\right|$ & $0.493 \mathrm{~d}-02$ & $0.101 \mathrm{~d}-01$ & $0.231 \mathrm{~d}-02$ & $0.252 \mathrm{~d}-02$ \\
\hline
\end{tabular}

TABLE 4. Maximum error for mesh $\mathrm{C}$

\begin{tabular}{|c|c|c|c|c|}
\hline$N$ & 0 & 1 & 3 & 5 \\
\hline $\max \left|u_{i}-u_{N, i}^{h}\right|$ & $0.409 \mathrm{~d}-01$ & $0.568 \mathrm{~d}-02$ & $0.433 \mathrm{~d}-03$ & $0.294 \mathrm{~d}-03$ \\
\hline $\max \left|v_{i}-v_{N, i}^{h}\right|$ & $0.408 \mathrm{~d}-02$ & $0.816 \mathrm{~d}-02$ & $0.624 \mathrm{~d}-03$ & $0.840 \mathrm{~d}-03$ \\
\hline
\end{tabular}

about $3.2 \%$ for mesh A, $1 \%$ for mesh B, and $0.25 \%$ for mesh C. The maximum norm of $v$ is about 0.555 , hence the maximum relative error for $v$ is about $11.7 \%$ for mesh A, $4.54 \%$ for mesh B, and $1.51 \%$ for mesh C. The convergence is fast; in fact, the rates are much higher than linear.

Table 2 shows the maximum of the errors $u-u_{N}^{h}$ and $v-v_{N}^{h}$ for mesh A when $N=1,3$, and 5; Tables 3-4 show the analogous results for meshes $\mathrm{B}$ and C. As we can see from the tables, for $u, N=3$ is good enough for meshes $\mathrm{A}$ and $\mathrm{B}$, since the meshes are too coarse and then the main errors are due to the coarse meshes. This becomes clear when the mesh is refined, $N=5$ did improve the accuracy for mesh C. For $v$, the effects of $N$ were not so significant as for $u$ for meshes $\mathrm{A}$ and $\mathrm{B}$; this is because on the boundary $\Gamma_{e}, v$ is very close to zero, so even for $N=0$, the error is already small. The effects of $N$ showed up only for finer meshes, as is shown in Table 4 for mesh C.

Figures 3-5 show the results for $u$ and $v$ along some curves, where the interior points are the points along the curve $A B C D E$ shown in Figure 2, and the boundary points are the points along the boundary $\Gamma_{e}$, i.e., the circumference with radius 2. The effects of $N$ are shown for meshes A-C; as shown in the figures, $N=5$ gives good approximations, and therefore in the computation very few terms in the bilinear form $B_{N}(u, v ; \tilde{u}, \tilde{v})$ are needed in order to get good accuracy. 


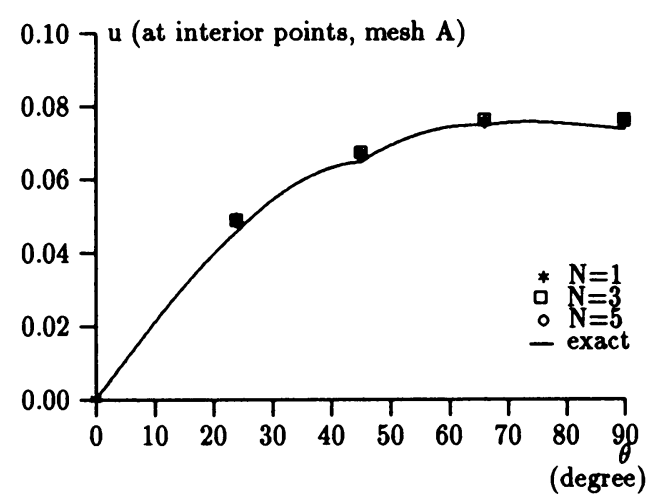

FIGURE 3

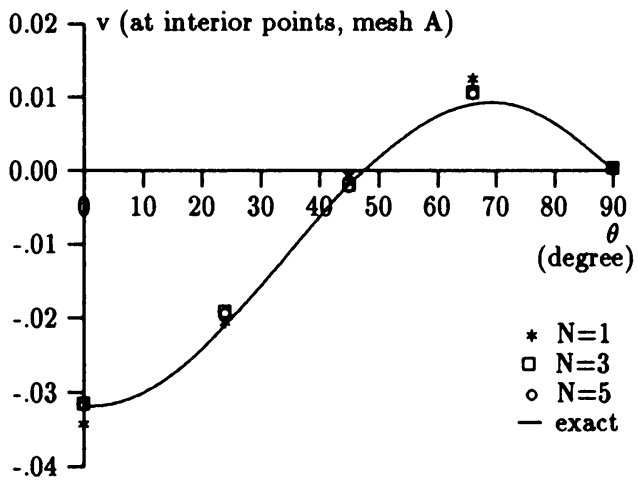

FiguRE 5



FIGURE 7

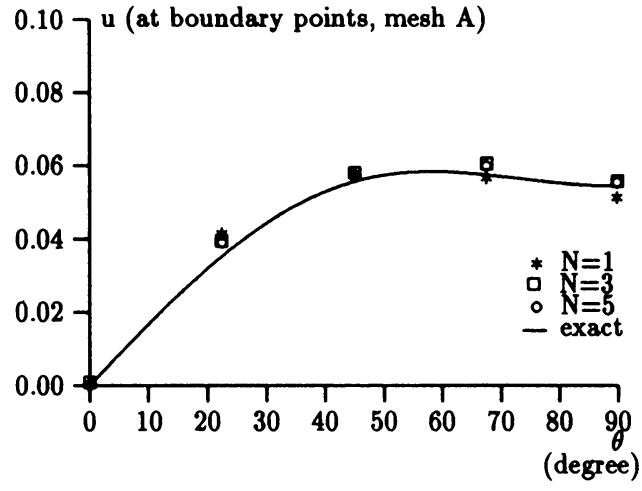

Figure 4

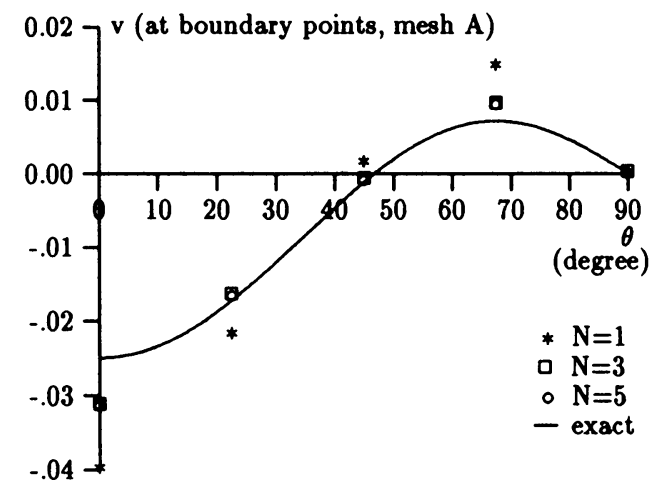

FigURE 6

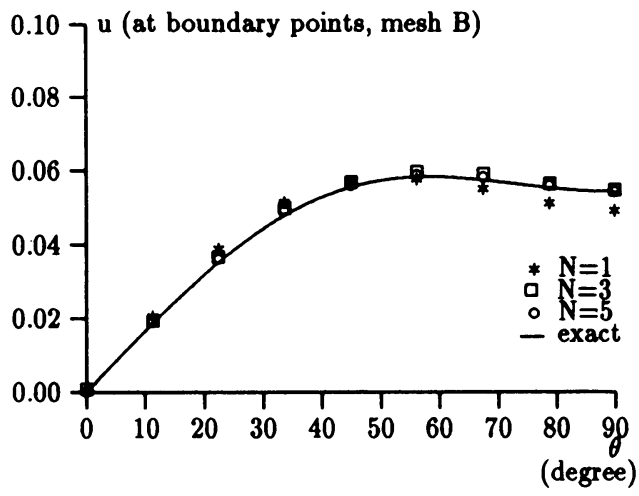

FigURE 8 


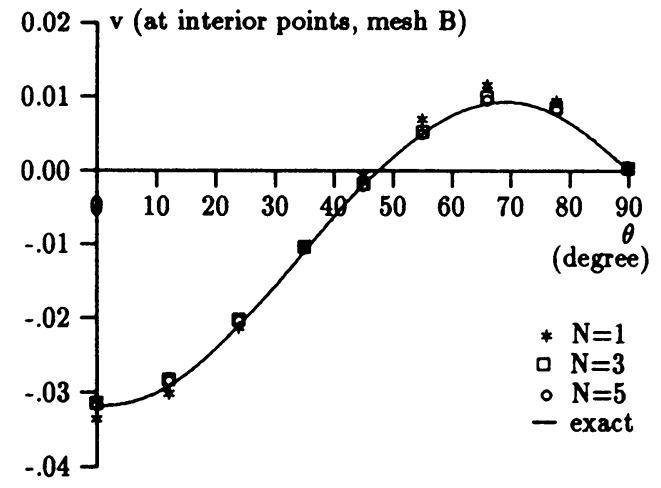

FIGURE 9

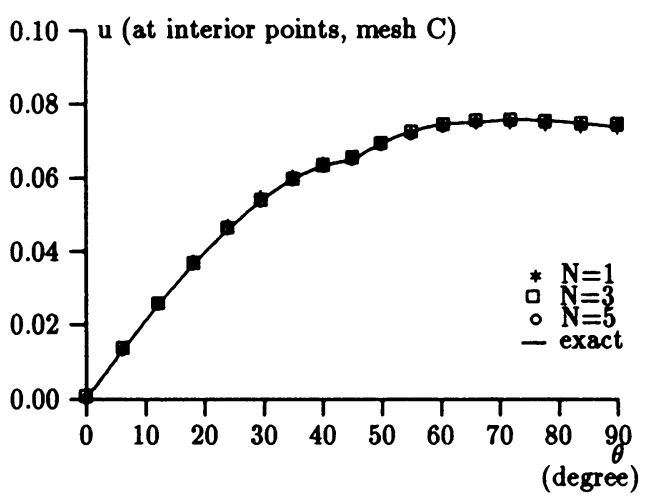

FIGURE 11

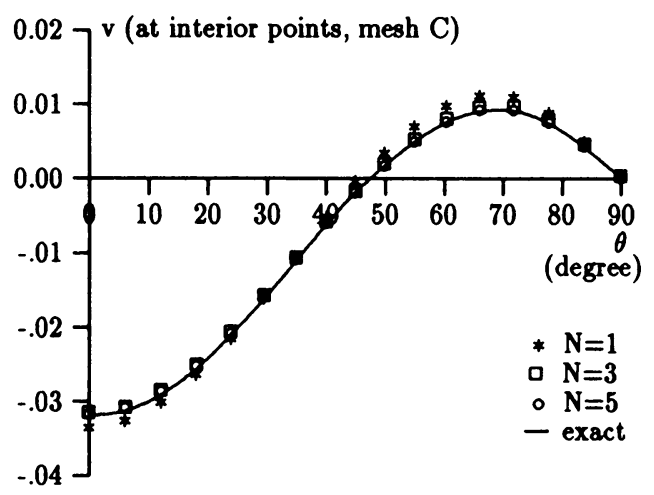

FIGURE 13

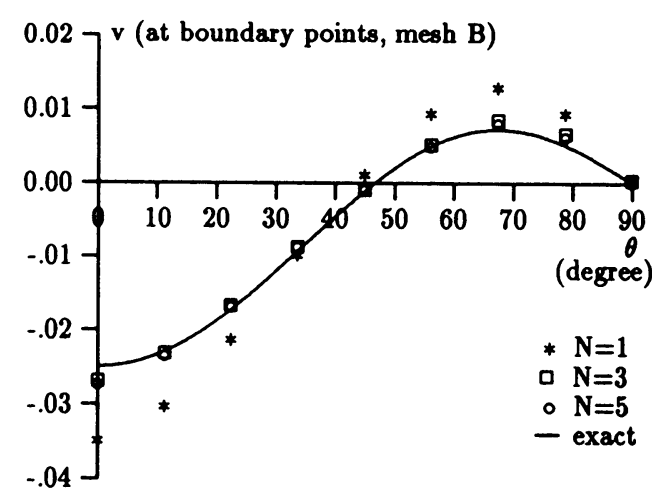

FIGURE 10

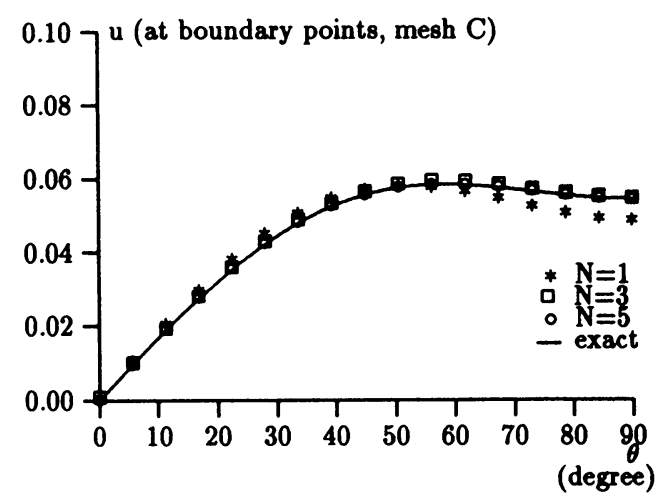

FIGURE 12

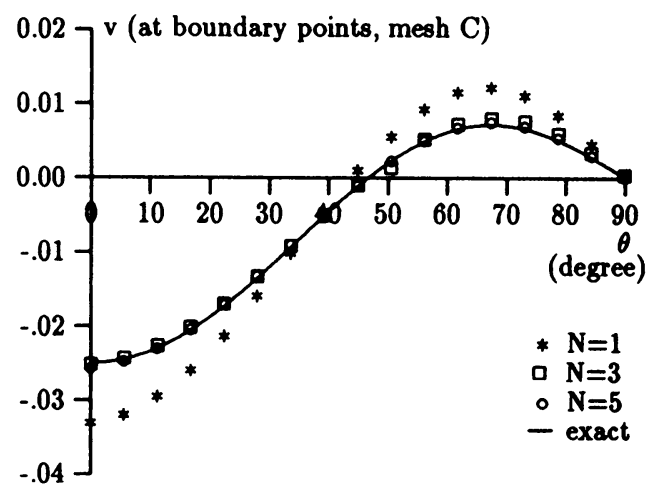

Figure 14 


\section{BIBLIOGRAPHY}

1. I. Babuška and A. K. Aziz, Survey lecture on the mathematical foundations of the finite element method, The Mathematical Foundations of the Finite Element Method with Applications to Partial Differential Equations (A. K. Aziz, ed.), Academic Press, New York, 1972, pp. 5-359.

2. P. G. Ciarlet, The finite element method for elliptic problems, North-Holland, Amsterdam, 1978.

3. C. I. Goldstein, A finite element method for solving Helmholtz type equations in waveguides and other unbounded domains, Math. Comp. 39 (1982), 309-324.

4. K. Feng, Asymptotic radiation conditions for reduced wave equation, J. Comput. Math. 2 (1984), 130-138.

5. T. M. Hagstrom and H. B. Keller, Exact boundary conditions at an artificial boundary for partial differential equations in cylinders, SIAM J. Math. Anal. 17 (1986), 322-341.

6. ___ Asymptotic boundary conditions and numerical methods for nonlinear elliptic problems on unbounded domains, Math. Comp. 48 (1987), 449-470.

7. $\mathrm{H}$. Han and $\mathrm{X}$. Wu, Approximation of infinite boundary condition and its application to finite element methods, J. Comput. Math. 3 (1985), 179-192.

8. A. Jepson and H. B. Keller, Boundary value problems on semi-infinite intervals. I. Linear problems, Numer. Math. (to appear).

9. H. B. Keller, Numerical solution of two point boundary value problems, No. 24, CBMS/NSF Regional Conf. Ser. in Appl. Math., SIAM, Philadelphia, PA, 1976.

10. M. Lenoir and A. Tounsi, The localized finite element method and its application to the two-dimensional sea-keeping problem, SIAM J. Numer. Anal. 25 (1988), 729-752.

11. M. Lentini and H. B. Keller, Boundary value problems on semi-infinite intervals and their numerical solution, SIAM J. Numer. Anal. 17 (1980), 577-604.

12. S. G. Mikhlin, The problem of the minimum of a quadratic functional, Holden-Day, San Francisco, London, Amsterdam, 1965.

13. N. I. Muskhelishvili, Some basic problems of the mathematical theory of elasticity, Noordhoff, Groningen, 1963.

14. J. L. Lions, Optimal control of systems governed by partial differential equations, SpringerVerlag, Berlin and Heidelberg, 1971.

Department of Applied Mathematics, Tsinghua University, Beijing, 100084, People's RePublic OF China

Current address: Department of Mathematics, University of Maryland, College Park, Maryland 20742

E-mail address: houdehan@olgao.umd.edu

Department of Mathematics, Carnegie-Mellon University, Pittsburgh, Pennsylvania 15213

Current address: Department of Mathematics, University of North Carolina at Charlotte, Charlotte, North Carolina 28223

E-mail address: xwu@unccvax.uncc.edu 